\title{
Prevalence And Clinical Significance Of Oncogenic CD79B And MYD88 Mutations In Primary Testicular Diffuse Large B-Cell Lymphoma: A Retrospective Study In China
}

\author{
Yan-ping Chen ${ }^{1,2, *}$ \\ Long-feng $\mathrm{Ke}^{3, *}$ \\ Jian-ping Lu' \\ Jian-chao Wang ${ }^{1,2}$ \\ Wei-feng Zhu' \\ Fang-fang Chen ${ }^{3}$ \\ Shao-feng Lin ${ }^{4}$ \\ Chun-wei $\mathrm{Xu}$ iD $^{3}$ \\ Mei-juan $\mathrm{Wu}^{5}$ \\ Gang Chen ${ }^{\mathrm{I}, 2}$
}

'Department of Pathology of Fujian Cancer Hospital, Fujian Medical University Cancer Hospital, Fuzhou 3500 I4, People's Republic of China; ${ }^{2}$ Department of Fujian Provincial Key Laboratory of Tumor Biotherapy, Fuzhou, Fujian 350014, People's Republic of China; ${ }^{3}$ Department of Molecular Pathology, Fujian Cancer Hospital, Fujian Medical University Cancer Hospital, Fuzhou, Fujian 3500I4, People's Republic of China; ${ }^{4}$ Department of Thoracic Surgery, Fujian Cancer Hospital, Fujian Medical University Cancer Hospital, Fuzhou 3500I4, People's Republic of China; ${ }^{5}$ Department of Pathology of Zhejiang Cancer Hospital, Hanzhou 310022, People's Republic of China

*These authors contributed equally to this work

Correspondence: Gang Chen No. 420, Fuma Road, Fuzhou, Fujian Province 3500I4, People's Republic of China

Tel/Fax +86 59l-83660063

Email naichengang@I26.com

Mei-juan Wu

No. I East Banshan Road, Gongshu

District, Hangzhou, Zhejiang 310022 ,

People's Republic of China

$\mathrm{Tel} / \mathrm{Fax}+86$ 57I-88I28257

Email wumj@zjcc.org.cn
This article was published in the following Dove Press journal: OncoTargets and Therapy

Purpose: In this study, we investigated the prevalence of $C D 79 B$ and $M Y D 88$ mutations and their relation to clinical characteristics in a cohort of Chinese patients with primary testicular diffuse large B cell lymphoma (PT-DLBCL).

Patients and methods: We examined the mutational status of $C D 79 B$ and $M Y D 88$ by Sanger sequencing, and the gene amplification and protein expression of MYD88 in tissue samples from 30 cases of PT-DLBCL by quantitative polymerase chain reaction and immunohistochemistry, respectively. Western blotting was used to analyze phosphorylated STAT3 (p-STAT3) and phosphorylated p65 (p-p65) protein expression in cell lines harboring retroviral constructs for WT MYD88 or MYD88 mutant.

Results: Immunophenotypically, MYD88 protein staining was positive in 26/30 $(86.67 \%)$ cases, and 23/30 (76.7\%) cases tested positive for p65 in the nucleus. Genetically, $C D 79 B$ mutation was found in $13 / 30(43.3 \%)$ cases, whereas the $M Y D 88^{\mathrm{L} 265 \mathrm{P}}$ mutation was found in $18 / 30(60.0 \%)$ cases. Interestingly, $C D 79 B$ and $M Y D 88$ mutations were more prevalent in the non-germinal center B cell (GCB) subtype ( $83.3 \%$ and $76.9 \%$, respectively) and were relatively rare in the GCB subtype (16.7\% and $23.1 \%$, respectively). Furthermore, although MYD 88 was significantly amplified in PT-DLBCL, the amplification status showed no correlation with its mutational status and protein expression. Clinicopathological comparison between the mutant and wild-type group showed that both $C D 79 B$ mutation and $M Y D 88^{\mathrm{L} 265 \mathrm{P}}$ were not significantly correlated with age, anatomical site, Ann Arbor stage, non-GCB/GCB subtype, p65 protein expression, BCL-2 protein expression, or BCL-2/c-MYC double expression $(P>0.05)$. Survival analyses showed that high IPI and advanced stage (stage III-IV) associated with worse outcome $(P<0.05)$. The expression of $\mathrm{p}$-STAT3 and p-p65 protein was upregulated in the mutant group, indicating that MYD 88 mutant activated NF- $\mathrm{kB}$ and JAK-STAT3 signaling.

Conclusion: Our results suggest that $M Y D 88$ and $C D 79 B$ mutations are important drivers of immune-privileged site-associated DLBCL and highlight potential therapeutic targets for personalized treatment.

Keywords: primary testicular lymphoma, $C D 79 B, M Y D 88$, mutation, diffuse large B cell lymphoma, gene amplification

\section{Introduction}

Primary non-Hodgkin's lymphoma of the testis (PTL) is an uncommon disease, accounting for approximately $9 \%$ of all testicular neoplasms and $1 \%-2 \%$ of all non-Hodgkin's lymphomas. ${ }^{1,2}$ PTL is the most common testicular malignancy in men older than 50 
years, presenting as focal masses with epididymal and scrotal involvement. ${ }^{3}$ Diffuse large B-cell lymphoma (DLBCL) is the most common histotype in primary forms, accounting for $80 \%-90 \%$ of cases. ${ }^{4}$ PT-DLBCLs that arise in sites previously considered to be immune sanctuaries show inferior responses to therapies. In the largest series of PTL patients reported to date, the outcome was very poor with a 5- and 10-year overall survival (OS) of $48 \%$ and $27 \%$, respectively ${ }^{5,6}$ The distinctive clinical and pathological characteristics of PT-DLBCL suggest that this entity might have a unique molecular and biological signature. ${ }^{7}$ However, the pathogenesis of PT-DLBCL is poorly understood.

Nuclear factor-kappa B $(\mathrm{NF}-\kappa \mathrm{B})$ is a transcription factor that regulates different cellular processes such as cell proliferation and survival and is normally transiently activated by antigen-dependent stimulation in $\mathrm{B}$ cells. ${ }^{8}$ Aberrant activation of NF- $\kappa \mathrm{B}$ is largely known to promote oncogenic characteristics such as anti-apoptosis, proliferation, and changes in cell adhesion. ${ }^{9,10}$ Oncogenic NF-кB activities have been demonstrated in various hematologic malignancies, including lymphoplasmacytic lymphoma, DLBCL, mucosa-associated lymphoid tissue lymphoma, multiple myeloma, Hodgkin lymphoma, and some leukemias. $^{11-15}$

In DLBCL, the mechanism of constitutive activation of the NF- $\mathrm{BB}$ pathway has been traced to somatic genetic alterations in the upstream pathway components. ${ }^{16}$ A recent gene expression profile-based molecular classification of DLBCL distinguished two main subtypes: activated $\mathrm{B}$-cell-like $(\mathrm{ABC})$ lymphoma and germinal center B cell (GCB)-like lymphoma. ${ }^{17}$ ABC-DLBCLs exhibit increased baseline NF- $\mathrm{B}$ activity and more frequent genetic alterations of the chronic active $\mathrm{B}$-cell receptor (BCR) signaling pathway components, including mutations of $C A R D 11$ and/or $C D 79 B$, and the Toll-like receptor (TLR) signaling pathway component myeloid differentiation primary response gene 88 (MYD88). ${ }^{15,18-20}$ These alterations are only detected in a subset of ABC-type DLBCL cases (MYD88, 29\%; CD79B, 18\%; and CARD11, 10\%). ${ }^{15}$ However, recent studies indicate that the prevalence of oncogenic MYD88 mutations varies greatly among ABC-DLBCLs presenting at different anatomical sites. $^{15,21,22}$ Interestingly, MYD88 mutations were by far the most prevalent mutations detected in cases of immune-privileged site-associated DLBCL presenting in the central nervous system $(75 \%)$ and testis $(71 \%){ }^{23}$

With the advent of various targeted therapeutic agents acting on NF-אB-related pathways, ${ }^{24-26}$ knowledge of the frequency of individual NF-kB-affecting mutations and the clinicopathological impact of such mutations would be valuable. In particular, identification of molecules involved in BCR and MYD88 signaling can provide a genetic tool to identify patients that may benefit from personalized treatment targeting these pathways. However, previous studies on the $C D 79 B$ and MYD88 mutations in PT-DLBCL are limited ${ }^{23,27,28}$ and the clinical significance of $M Y D 88$ mutations remains unclear. Furthermore, the mutational status of $C D 79 B$ and $M Y D 88$ in Chinese PT-DLBCL patients has not been elucidated. In this study, we examined the prevalence, clinicopathologic characteristics, and prognosis of $C D 79 B$ and MYD88 mutations in a cohort of Chinese patients with PT-DLBCL.

\section{Materials And Methods}

\section{Patient Samples}

This retrospective study was performed on 30 patients with PT-DLBCL originally diagnosed and treated at Fujian Cancer Hospital and Zhejiang Cancer Hospital, China between January 2003 and June 2016. According to the literature, ${ }^{29}$ primary testicular lymphoma was defined as a lymphoma that presented with a clinically dominant and primary testicular mass, and also allowed the inclusion of patients who were ultimately shown to have advanced-stage disease. When the testis became involved after systemic lymphoma was diagnosed, the lymphoma was regarded as a secondary testicular lymphoma. Cases of secondary testicular DLBCL were excluded from the selection. Three expert pathologists reviewed all cases according to the 2017 World Health Organization classification of tumors of hematopoietic and lymphoid tissues. ${ }^{30}$ All patients underwent orchidectomy with or without chemotherapy. The first-line chemotherapy regimen was mostly cyclophosphamide, doxorubicin, vincristine, prednisolone (CHOP), or rituximab plus $\mathrm{CHOP}$ (R-CHOP). Ten samples of normal lymph nodes derived from patients visiting Fujian Cancer Hospital were collected and considered as a control group. This study was carried out in accordance with the Declaration of Helsinki and written informed consent was obtained from the patients or their legal guardians. The study protocol was approved by the institutional review boards of Fujian Cancer Hospital and Zhejiang Cancer Hospital.

\section{Immunohistochemistry Analysis}

Immunohistochemical analysis was performed using fully automated protocols on a Bond-III Autostainer (Leica 
Biosystems, Melbourne, Australia). Four-micrometer-thick sections of formalin-fixed paraffin-embedded (FFPE) tissue were subjected to staining protocols with the following antibodies: CD20, CD3, CD10, BCL-6, MUM-1, Ki-67, BCL-2, MYC, p65, and MYD88. Detailed information regarding the primary antibodies and their sources, dilution ratios, clones, and cut-off values are shown in Table 1. Germinal center B cell (GCB) subtype of DLBCL and non-GCB subtype of DLBCL were classified based on immunohistochemical staining of CD10, BCL-6, and MUM-1 by Hans' algorithm. ${ }^{31} \mathrm{We}$ used a scoring system for MYD88 expression according to previous reports. $^{27,32}$ The staining intensity of the cytoplasm was scored as either 0 (negative), 1 (weak), 2 (moderate), or 3 (intense), and the extent of staining was scored as either $0(0 \%$ of the tumor area stained), $1(<10 \%), 2(10 \%-50 \%)$, or 3 $(>50 \%)$. These two parameters were combined to reach a final score ranging from 0 to 6 . Final scores of 5 or 6 were classified as high MYD88 expression and scores of 0-4 were classified as low expression.

\section{CD79B And MYD88 Mutation Analysis}

Genomic DNA was isolated from three pieces of a 5- $\mu$ m-thick section of the FFPE tumor tissue using the QIAamp FFPE DNA Tissue Kit (Qiagen, Germantown, MD, USA) according to the manufacturer's instructions. PCR was carried out using AmpliTaq Gold polymerase (Applied Biosystems, Weiterstadt, Germany) according to the manufacturer's instructions under the following conditions: $94^{\circ} \mathrm{C}$ for $5 \mathrm{~min}$ followed by 34 cycles of denaturation for $30 \mathrm{~s}$ at $94^{\circ} \mathrm{C}$, annealing for $30 \mathrm{~s}$ at $60^{\circ} \mathrm{C}$, and extension for $45 \mathrm{~s}$ at $72^{\circ} \mathrm{C}$. The primer pairs for $C D 79 B$ (exon 5) and $M Y D 88$ (exon 5) were designed using Primer-BLAST as follows: $C D 79 B-5 \mathrm{~F}$

Table I Detailed Information Of Primary Antibodies And Their Cut-Off Values

\begin{tabular}{|c|c|c|c|c|}
\hline Antibody & Source & Clone & Dilution & $\begin{array}{l}\text { Cut-Off } \\
\text { Value }\end{array}$ \\
\hline $\mathrm{CD} 20$ & $\begin{array}{l}\text { MXB } \\
\text { Biotechnologies }\end{array}$ & L26 & Ready-to-use & \\
\hline CD3 & $\begin{array}{l}\text { MXB } \\
\text { Biotechnologies }\end{array}$ & SP7 & Ready-to-use & \\
\hline CDIO & Dako & $56 \mathrm{C} 6$ & Ready-to-use & $30 \%$ \\
\hline $\mathrm{BCL}-6$ & Dako & PG-B6p & Ready-to-use & $30 \%$ \\
\hline MUM-I & Dako & MUMIP & Ready-to-use & $30 \%$ \\
\hline $\mathrm{Ki}-67$ & Dako & MIB-I & Ready-to-use & \\
\hline $\mathrm{BCL}-2$ & Dako & 124 & Ready-to-use & $70 \%$ \\
\hline MYC & Abcam & Y69 & $1: 500$ & $40 \%$ \\
\hline p65 & Santa Cruz & sc- 109 & $1: 300$ & $30 \%$ \\
\hline MYD88 & Abcam & EPR590(N) & $1: 100$ & \\
\hline
\end{tabular}

(5'-GGGCTGGGGGACACTAACACTC-3'), CD79B-5R (5'TGGGTGCTCACCTACAGACCAC-3'), MYD88-5F (5'-AA CCCTGGGGTTGAAGACTG-3'), and MYD88-5R (5'-AG TCTTCAGGGCAGGGACAA-3'). The PCR products were routinely purified and sequenced in both directions using the BigDye Terminator version 3.1 Cycle Sequencing Kit (Applied Biosystems, Foster City, CA, USA). At least two independent PCR and sequencing experiments were conducted to confirm mutations, which were proven not to be single nucleotide polymorphisms via reference to the National Center for Biotechnology Information dbSNP database.

\section{MYD88 Copy Number Analysis}

Quantitative real-time PCR was performed to analyze the copy number status of $M Y D 88$ with normalization using the reference gene RPP30, a single copy gene encoding the ribonuclease P/MRP $30-\mathrm{kDa}$ subunit. ${ }^{27}$ The primer pairs used for MYD88 and RPP30 were as follows: MYD88F 5'-GATCCC CATCAAGTACAAGGCAA-3', MYD88R 5'-AAGGCGAG TCCAGAACCAAG-3', RPP30F 5'-GCCCGGCTCTATGAT GTTGT-3', and RPP30R 5'-TCCAGATGAGTCAAAAC AGGTCA-3'. The quantitative PCR was carried out using Stratagene Mx3000P (Agilent, Germany) according to the manufacturer instructions. In brief, $20 \mathrm{ng}$ of genomic DNA from the FFPE tissue and $300 \mathrm{nM}$ of primer in a final volume of $20 \mu \mathrm{L}$ with SsoAdvanced Universal SYBR Green Supermix (Bio-Rad) was amplified under the following thermal cycling conditions: enzyme activation for $3 \mathrm{~min}$ at $98^{\circ} \mathrm{C}$ followed by 45 cycles of denaturation for $15 \mathrm{~s}$ at $98^{\circ} \mathrm{C}$, and annealing, extension, and plate reading for $30 \mathrm{~s}$ at $60^{\circ} \mathrm{C}$. Because the estimated PCR efficacies of MYD88 and RPP30 were almost identical and very close to $100 \%(94.6 \%$ and $95.1 \%$, respectively), we quantified the $M Y D 88$ copy number (MYD88/RPP30) using the $\triangle \mathrm{CT}$ method. ${ }^{27}$

\section{Statistical Analysis}

SPSS software (version 19.0, IBM, Armonk, NY, USA) was used for all statistical analyses. The patient characteristics were compared across different subgroups using Fisher's exact test. Correlation analysis of the categorical variables was analyzed using the chi-squared test. OS was determined from the date of diagnosis to the date of death caused by lymphoma or termination of follow-up. Univariate analysis and survival curves were generated by the Kaplan-Meier method and compared using the log rank test. Multivariate analysis was conducted with the Cox proportional hazards model. A $P$-value of $<0.05$ was considered statistically significant. 


\section{Cell Culture, Retroviral Constructs, And Transduction, And Western Blotting}

TMD8 and LY1 cell lines were cultured in RPMI 1640 medium containing 10\% FBS (Gibco, Carlsbad, CA, USA) and $100 \mathrm{U} / \mathrm{mL}$ of penicillin and streptomycin (Invitrogen, Carlsbad, CA, USA). Cells were maintained at $37^{\circ} \mathrm{C}$ in $5 \%$ $\mathrm{CO}_{2}$ in a humid atmosphere.

Point mutations were made to generate $M Y D 88^{\mathrm{L} 265 \mathrm{P}}$. MYD88 mutants were created with the Phusion site-directed mutagenesis kit according to the manufacturer's instructions (New England BioLabs), using vLyt2-MYD88-EGFP vector as templates. All cDNA inserts from PCR cloning and sitedirected mutagenesis were verified by sequencing. The MYD88 mutagenesis primers were as follows: L265P forward P-CATCAGAAGCGACCGATCCCCATCAAG and L265P reverse $\mathrm{P}$ GGCACCTGGAGAGAGGCTGAGTGCAAA. For transfer into a retroviral vector system, $M Y D 88^{\mathrm{WT}}$ and $M Y D 88^{\mathrm{L} 265 \mathrm{P}}$ were amplified by polymerase chain reaction using Phusion polymerase (New England Biolabs) according to the manufacturer's recommendations.

HEK293T cells $(150,000$ cells per well of a 6-well plate) were transfected using Lipofectamine 2000 (Invitrogen) with $1 \mu \mathrm{g}$ to $5 \mu \mathrm{g}$ of plasmids. Stable cell lines were made by transfection of HEK293T cells with the respective plasmids and then selection with blasticidin (Sigma-Aldrich) for 1 week. Pools of stable lines expressing GFP alone or GFPtagged proteins were used for the experiments.

Whole cell lysates were subjected to $4 \%-12 \%$ sodium dodecyl sulfate-polyacrylamide gel electrophoresis and transferred to polyvinylidene fluoride (PVDF) membranes (Invitrogen), followed by incubation with primary antibodies. The following antibodies were used: anti-phospho p65 and anti-phospho STAT3 (Tyr705) (Cell Signaling Technology, Denvers, MA, USA).

\section{Results}

\section{Clinical Characteristics}

The study group included 30 patients with PT-DLBCL. The patients' ages at diagnosis ranged from 38 to 85 years with a median age of 62 years. Seventeen patients (56.7\%) were older than 60 years. The main symptoms of the PTDLBCL (painless testicular mass) were initially present on the left side in 12 patients $(40 \%)$, right side in 15 patients $(50 \%)$, and bilaterally in three patients $(10 \%)$. Complete clinical information was recorded for all patients. B symptoms were identified in four (13.3\%) patients. There were five $(16.7 \%), 13(43.3 \%)$, five $(16.7 \%)$, and seven (23.3\%) patients with Ann Arbor stages I, II, III, and IV disease, respectively. Furthermore, there were 21 $(70.0 \%)$ and nine $(30.0 \%)$ patients with low International Prognostic Index (IPI) and high IPI, respectively. Clinical outcomes were available for 26 patients included in the study. At the time of the last clinical follow-up (range, 4-56 months), six (23.1\%) patients had died, and the median OS was 24 months. The 3-year OS rate was $73.20 \%$.

\section{Morphological And Immunophenotypic Features}

A representative image of the histomorphology of PTDLBCL is shown in Figure 1. All orchidectomy specimens showed diffuse proliferation of large neoplastic lymphocytes. A seminiferous tubule entrapped by lymphoma cell infiltration was also present. Atypical large lymphocytes had large irregular nuclei with one or more prominent nucleoli. A high mitotic rate and atypical mitoses were common findings in most cases.

All cases were strongly positive for $\mathrm{CD} 20$ and negative for CD3. According to Hans's algorithm, 26/30 (86.7\%) cases demonstrated a non-GCB immunophenotype, whereas 4/30 (13.3\%) cases demonstrated a GCB immunophenotype. The ratio of GCB to non-GCB phenotype was 1:6.5. The Ki67 proliferation rate was generally high, with a median of $60 \%$ and a range of $50 \%-90 \%$. There were $25 / 30(83.3 \%)$ and $13 / 30(43.3 \%)$ cases that tested positive for BCL-2 and MYC, respectively, and 11/30 (36.7\%) cases were positive for both BCL2 and MYC (double expression). Twenty-three (76.7\%) cases expressed the NF- $\mathrm{kB}$ subunit p65. The MYD88 protein was expressed in 26/30 (86.67\%) cases with high degrees of intensity (Figure 1). The expression pattern of MYD88 exhibited no correlation with the patient's age $(P=1.000)$, location $(P=0.406)$, Ann Arbor stage $(P=0.913)$, and non-GCB/GCB subtype $(P=0.65)$ (Table 2$)$. However, the MYD88 protein stain was significantly associated $(P=0.037)$ with MYD 88 missense mutation (Table 3 ).

\section{Mutational Status Of CD79B And MYD88 And Clinicopathological Associations}

With respect to the mutation status, $C D 79 B$ exon 5 and MYD88 exon 5 were successfully amplified in all samples. At least one mutation of the two genes analyzed was detected in 22/30 (73.3\%) cases, with 18/30 (60.0\%) cases showing MYD88 mutation and 13/30 (43.3\%) cases showing $C D 79 B$ mutation. All 13 patients who harbored 


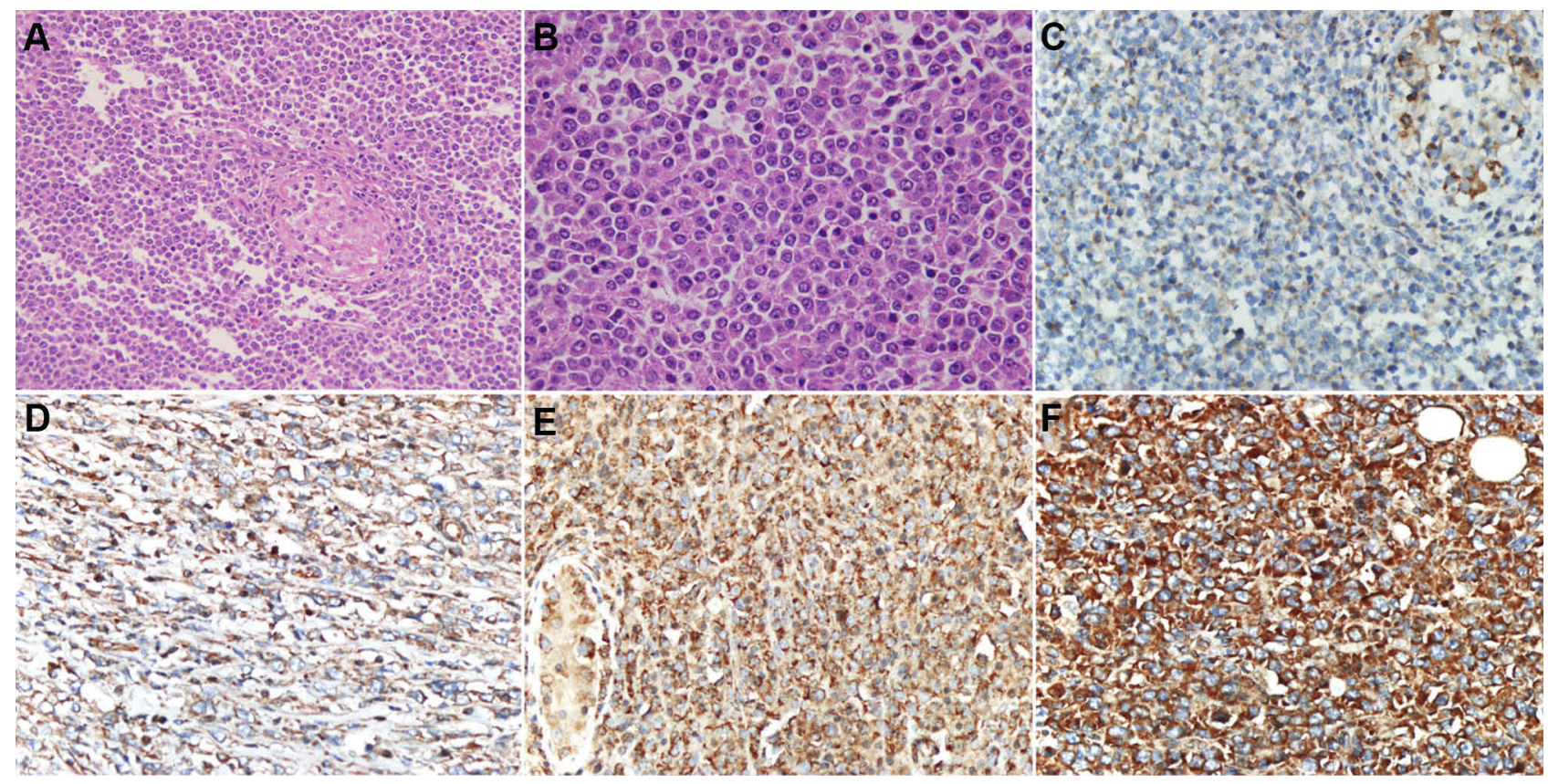

Figure I Representative hematoxylin and eosin (H\&E) staining of PT-DLBCL and immunostaining of MYD88. (A) Orchidectomy specimens showing diffuse proliferation of large neoplastic lymphocytes (H\&E, $\times 200)$. (B) Atypical large lymphocytes had large irregular nuclei with one or more prominent nucleoli (H\&E, $\times 400)$. MYD88 is expressed in the cytoplasm of lymphoma cells. Intensity scoring of MYD88 immunohistochemistry: (C) 0, negative; (D) I, weak; (E) 2, moderate; and (F) 3, intense ( $\times 400)$.

CD79B mutations showed missense mutations. The main affected position (Y196) was the first tyrosine of the immunoreceptor tyrosine-based activation motif (ITAM) domain (Figure 2). The overall frequency of mutation was higher in our cohort than reported previously. All 18 MYD88 mutations were L265P, an evolutionarily invariant residue in the hydrophobic core of the Toll/interleukin (IL)-1 (TIR) receptor domain of MYD88 (Figure 2). The $M Y D 88^{\mathrm{L} 265 \mathrm{P}}$ mutation coexisted with a $C D 79 B$ mutation in eight cases, which constituted $61.5 \%$ of $C D 79 B$ mutations identified and $44.4 \%$ of $M Y D 88^{\mathrm{L} 265 \mathrm{P}}$ cases. Intriguingly, $C D 79 B$ and $M Y D 88$ mutations were more prevalent in the non-GCB subtype $(83.3 \%$ and $76.9 \%$, respectively) and were relatively uncommon in the GCB subtype (16.7\% and $23.1 \%$, respectively). The MYD88 and $C D 79 B$ mutational status showed no significant correlation with the patient's age, location, Ann Arbor stage, IPI, and non-GCB/GCB subtype (Table 2).

\section{Amplification Status Of MYD88}

In quantitative real-time PCR analysis, MYD 88 was successfully amplified in all cases. The copy number of MYD 88 in the PT-DLBCL cases ranged from 1.11 to 22.76 with a median of 4.19. Compared with the level detected in normal lymph nodes (range, 0.86-1.24; median, 1.005), the MYD88 gene showed significant enhancement of amplification in PT-
DLBCL cases $(P<0.01)$. However, there was no significant difference in the amplification status between PT-DLBCL cases harboring the MYD 88 wild type (median, 4.5; range, 1.52-22.76) and L265P mutation (median, 4.355; range, 1.11-20.15) $(P=0.654)$. The amplification status (median, 4.67; range, 1.11-20.15) was not associated with the expression of MYD88 (median, 2.435; range, 1.62-22.76) $(P=0.141)$ (Table 3).

\section{Survival And Analysis Of Prognostic Factors}

Univariate analyses of the clinical, pathological, and genetic factors influencing the OS of patients with PT-DLBCL are summarized in Table 4. Univariate analysis showed that high IPI and advanced stage (stage III-IV) were associated with worse outcome $(P<0.001$ and $P=0.036$, respectively; Figure 3). $M Y D 88^{\mathrm{L} 265 \mathrm{P}}$ and BCL-2/c-MYC co-expression were marginally associated with a shorter $\mathrm{OS}(P=0.053$ and $P=0.055$, respectively). However, the $C D 79 B$ mutational status and expression level of MYD88 did not affect the prognosis ( $P=0.947$ and $P=0.719$, respectively). The factors most predictive of OS by univariate analysis were then included in the multivariate analysis. Because of the small sample size, no independent prognostic factor was associated with OS, although advanced stage $(P=0.071$, hazard ratio 
Table 2 Clinicopathologic Characteristics Of PT-DLBCL With CD79B And MYD88 Mutations And MYD88 Expression

\begin{tabular}{|c|c|c|c|c|c|c|c|}
\hline Parameter & No. Patients & CD79B Y I96 & $p$ & MYD88 L265P & $p$ & MYD88 & $p$ \\
\hline Location & & & 0.638 & & 0.880 & & 0.406 \\
\hline Left & 13 & 5 & & 8 & & 10 & \\
\hline Right & 17 & 8 & & 10 & & 16 & \\
\hline Age (years) & & & 0.599 & & 0.094 & & 1.000 \\
\hline$\leq 60$ & 12 & 4 & & 5 & & 10 & \\
\hline$>60$ & 18 & 9 & & 13 & & 16 & \\
\hline Ann Arbor stage & & & 0.638 & & 0.547 & & 0.913 \\
\hline I-II & 18 & 8 & & II & & 15 & \\
\hline III-IV & 12 & 5 & & 7 & & 11 & \\
\hline IPI score & & & 0.684 & & 0.792 & & 0.582 \\
\hline Low (0-2) & 21 & 9 & & 13 & & 19 & \\
\hline High (3-5) & 9 & 4 & & 5 & & 7 & \\
\hline Cell-of-origin & & & 0.406 & & 1.000 & & 0.913 \\
\hline $\mathrm{GCB}$ & 4 & 3 & & 3 & & 3 & \\
\hline Non-GCB & 26 & 10 & & 15 & & 23 & \\
\hline BCL-2 & & & 0.099 & & 1.000 & & 0.317 \\
\hline Positive & 26 & 13 & & 16 & & 22 & \\
\hline Negative & 4 & 0 & & 2 & & 4 & \\
\hline BCL-2/c-MYC & & & 0.838 & & 1.000 & & 0.970 \\
\hline Positive & 11 & 4 & & 7 & & 9 & \\
\hline Negative & 19 & 9 & & 11 & & 17 & \\
\hline P65 & & & 0.286 & & 0.792 & & 0.582 \\
\hline Positive & 23 & 12 & & 13 & & 19 & \\
\hline Negative & 7 & 1 & & 5 & & 7 & \\
\hline
\end{tabular}

$3.264,95 \%$ confidence interval $0.015-1.185$ ) showed a trend toward worse OS.

\section{MYD88 Mutant Activate NF- $\kappa B$ And JAK-STAT3 Signaling}

Western blot analysis of phosphorylated STAT3 (p-STAT3) and phosphorylated p65 (p-p65) protein expression in TMD8 and LY1 cell lines harboring retroviral constructs for WT MYD88 or MYD88 mutant are shown in Figure 4. Compared with the negative and blank controls, the expression of p-STAT3 and p-p65 proteins were significantly upregulated in the MYD88 mutant group, indicating that MYD88 mutant activated NF-kB and JAK-STAT3 signaling.

\section{Discussion}

DLBCL is the most common lymphoma subtype and accounts for $30 \%-40 \%$ of all cases of adult non-Hodgkin lymphoma. $^{30,33}$ DLBCL represents a class of clinically, immunophenotypically, and genetically homogeneous lymphomas, which have been subdivided into morphological variants, molecular and immunophenotypical subgroups, and distinct disease entities such as primary central nervous system lymphoma and primary mediastinal large B-cell lymphoma. According to the 2017 version of the World Health Organization classification of lymphoid malignancies, PTDLBCL is classified within the category of DLBCL, not otherwise specified. ${ }^{30}$ However, recent studies confirmed

Table 3 Correlation Between MYD88 Mutational Status, Amplification, And Its Protein Expression

\begin{tabular}{|l|l|l|l|l|l|l|l|}
\hline \multirow{2}{*}{ MYD88 } & No. Patients & \multicolumn{2}{|l|}{ MYD88 L265P } & \multirow{2}{*}{$\boldsymbol{p}$} & \multicolumn{2}{|l|}{ MYD88 Amplification } \\
\cline { 3 - 4 } & & Mutant & Wild Type & & Present & Absent \\
\hline Positive & 26 & 18 & 8 & 0.037 & 14 & 12 \\
Negative & 4 & 0 & 4 & & 0 & 4 \\
\hline
\end{tabular}


A

\section{G A C T/C G A T C C}

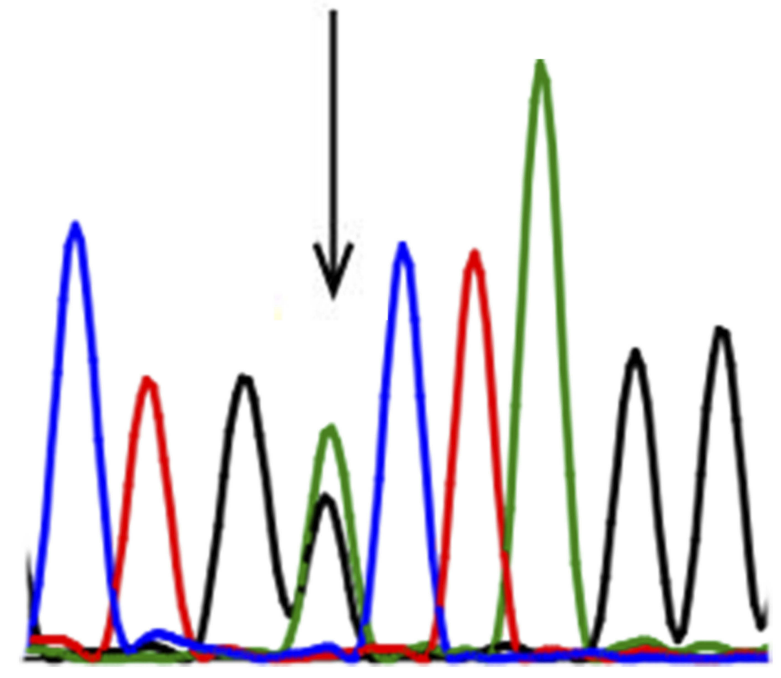

MYD88 c.794T $>C$

C

\section{A C C T/A A C G A}
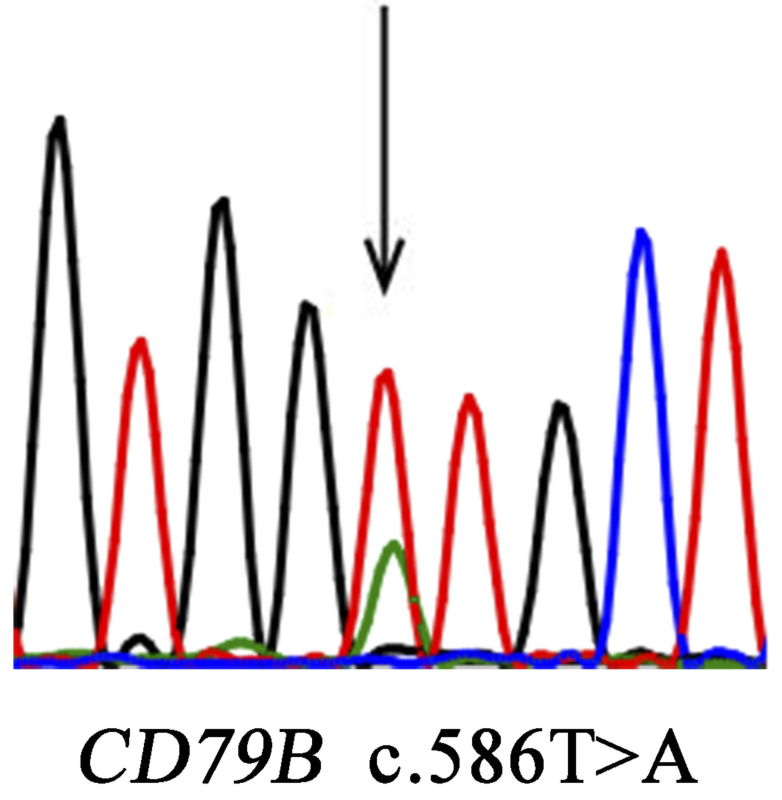

B
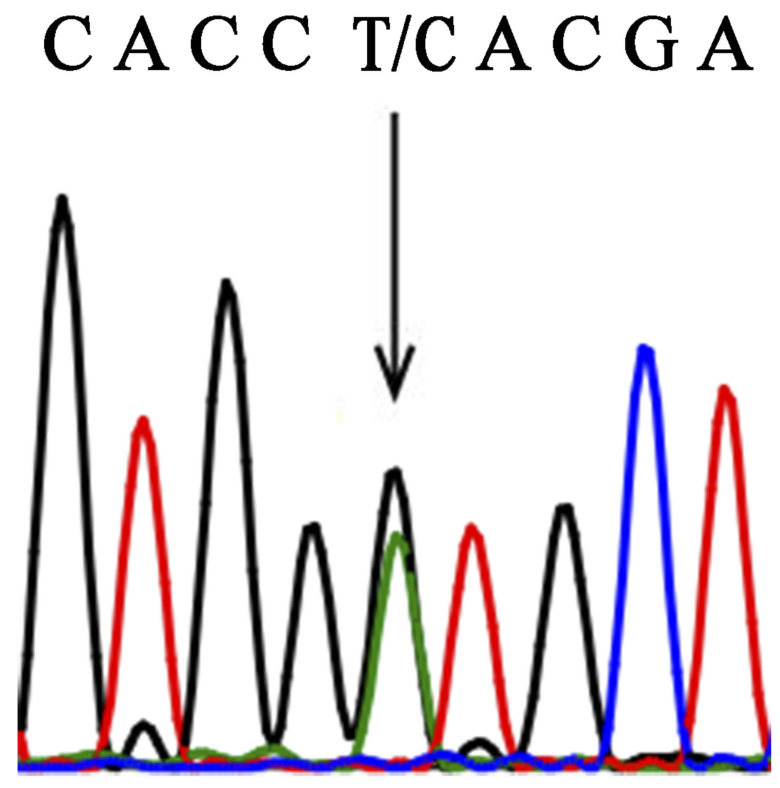

CD79B c.586T $>$ C

C A C C T A/C C G A

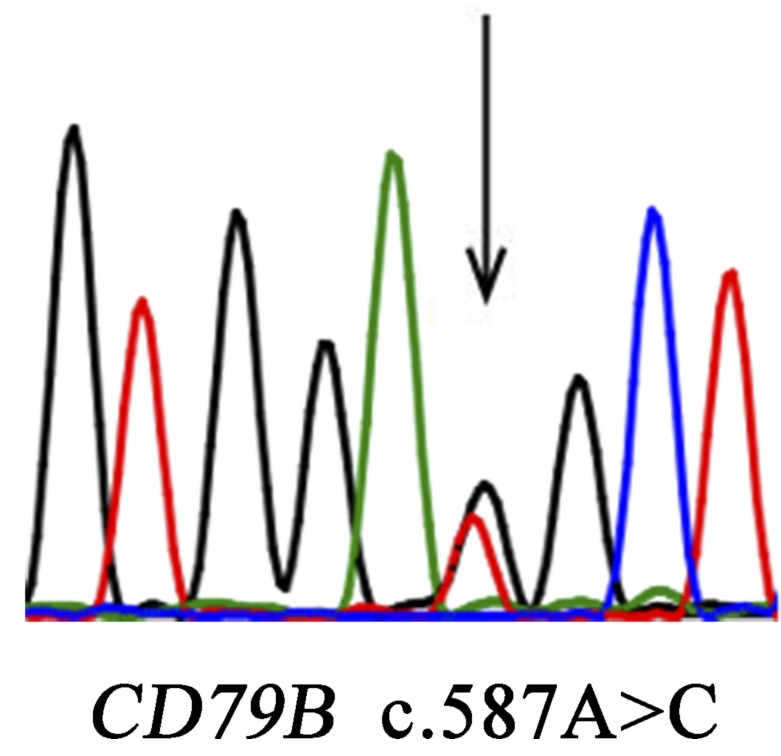

Figure 2 Representative results of Sanger sequencing. (A) MYD88 ${ }^{\mathrm{L} 265 \mathrm{P}}$. A CTG (leucine) codon was changed to a CCG (proline) codon. (B) CD79B. ${ }^{\text {I } 96 \mathrm{~N}}$ A TAC (tyrosine) codon was changed to a CAC (histidine) codon. (C) CD79B. ${ }^{\text {YI96N }}$ A TAC (tyrosine) codon was changed to an AAC (asparagine) codon. (D) CD79B ${ }^{\text {YI965 }}$. A TAC (tyrosine) codon was changed to a TCC (serine) codon.

the unique features of PT-DLBCL, including its prevalence in older males, high risk of relapse, propensity to involve the central nervous system, and poor outcome. The distinctive clinical and pathological characteristics of PT-DLBCL suggest that it represents a unique subtype of DLBCL. In the present study, clinicopathological factors, including patient 
Table 4 Univariate Analysis In 26 Patients With PT-DLBCL

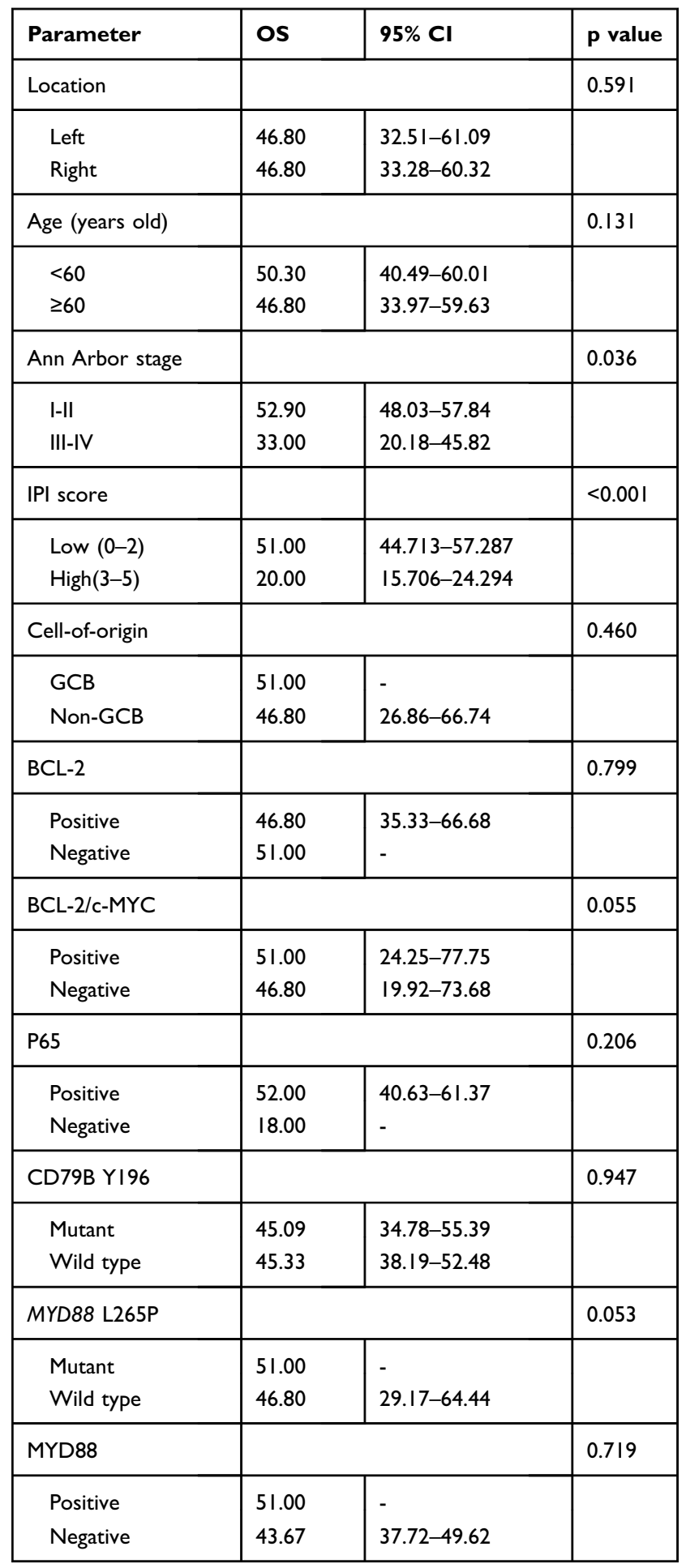

age, predominance of non-GCB subtype, and poor outcome, were in accordance with earlier studies.

Activation of the NF- $\mathrm{KB}$ pathway is a hallmark of ABCDLBCL. NF- $\kappa B$ activation might be triggered by stimulation of either BCR, tumor necrosis factor, or the TLR pathway. ${ }^{16}$ Somatic mutations affecting the ITAM signaling modules of $\mathrm{CD} 79 \mathrm{~B}$, and in some cases of $\mathrm{CD} 79 \mathrm{~A}$, were detected frequently in ABC-DLBCL cell lines and biopsy samples but rarely in other DLBCLs and never in Burkitt's lymphoma or mucosa-associated lymphoid tissue lymphoma. $C D 79 B$ mutations were shown to increase surface $\mathrm{BCR}$ expression and nullify the negative regulation of $\mathrm{BCR}$, which were suggested to support the "chronic active" BCR signaling that leads to constitutive NF- $\mathrm{kB}$ activation in ABC-DLBCL. It was initially reported that $C D 79 B$ mutations were present in $21 \%$ of ABC-DLBCL cases and $3 \%$ of GCB-DLBCL cases, although subsequent studies described a lower prevalence of only $8.5 \%-12.2 \% .{ }^{14,23}$ In the current study, we found that the $C D 79 B$ ITAM was mutated in cases of PT-DLBCL with a much higher prevalence of $43.3 \%$. In accordance with a previous study, ${ }^{28}$ these mutations were predominantly present in cases of non-GCB-type DLBCL, occurring in $83.3 \%$ of non-GCB-DLBCLs but only in $16.7 \%$ of GBC-DLBCLs.

MYD88 is an adaptor protein of TLR and IL-1 receptors, which mediates the TLR and IL-1R signaling pathways in the innate immune response. MYD88 activates the NF- $\mathrm{kB}$ pathway through association with IL-1 receptor-associated kinase (IRAK) 1 and IRAK4. ${ }^{7}$ Activating mutations in MYD 88 were initially identified in DLBCL by Ngo et al., ${ }^{8}$ who showed that the most frequent and most oncogenic form was the L265P mutation of the TIR domain, which was detected in $29 \%$ of ABC-DLBCL cases but was relatively rare in GCB-DLBCL. $M Y D 88^{\mathrm{L} 265 \mathrm{P}}$ forms a stable protein complex with IRAK1 and IRAK4 and promotes the constitutive activation of the NF- $\kappa$ B and JAK-STAT3 pathways, promoting malignant cell survival in these lymphomas. Our study demonstrated that the expression of p-STAT3 and p-p65 protein were upregulated in the mutant group, indicating that MYD88 mutant activated NF- $\mathrm{KB}$ and JAK-STAT3 signaling.

To date, MYD 88 mutations have been described in only $9 \%$ of gastric mucosa-associated lymphoid tissue lymphoma, $3 \%$ of chronic lymphocytic leukemia, $5 \%$ of Burkitt's lymphoma, and $29 \%$ of systemic ABC-DLBCL cases. ${ }^{7,8}$ Recent studies indicate that the prevalence of an oncogenic MYD 88 mutation varies greatly among ABC-DLBCLs presenting at different anatomical sites. ${ }^{15,21,22}$ Intriguingly, Kraan et al. ${ }^{23}$ found that MYD88 mutations were by far the most prevalent mutation detected in cases of immune privileged-DLBCL presenting in the central nervous system $(75 \%)$ or testis (71\%), and were relatively uncommon in nodal (17\%) and gastrointestinal tract lymphomas (11\%). We observed MYD $88^{\mathrm{L} 265 \mathrm{P}}$ mutations in $60.0 \%$ of the patients with PTDLBCL. The frequency of mutations was lower than those 

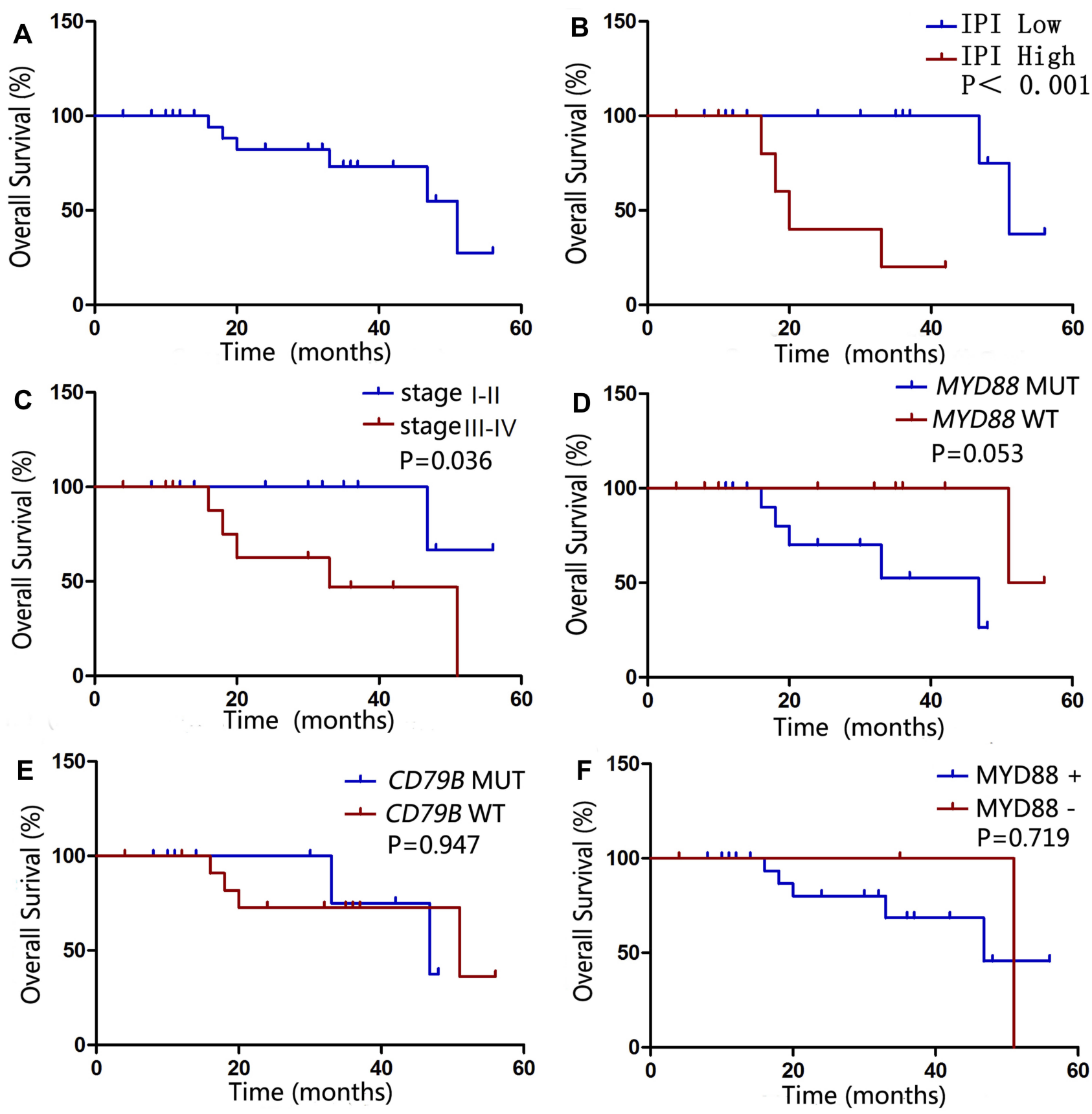

Figure 3 Univariate survival analysis of patients with PT-DLBCL. Overall survival (OS) of 26 patients with PT-DLBCL. (A). High IPI and advanced stage (stage III-IV) was associated with worse outcome $(P<0.05)$. (B and $\mathbf{C})$. However, the MYD88 (D) and CD79B (E) mutational status and expression level of MYD88 (F) did not affect the prognosis $(P>0.05)$.

reported by Kraan et al. ${ }^{23}$ and Oishi et al. ${ }^{27}(71 \%$ and $82 \%$, respectively); however, interestingly, the percentage of coexistence of this mutation with $C D 79 B$ mutations was much higher than those reported in previous studies. ${ }^{23,27,28}$

Our study confirms the previous finding that $M Y D 88$ and $C D 79 B$ mutations are predominant in ABC-DLBCL and often coexist within the same tumor. Moreover, MYD88 and $C D 79 B$ mutations are strongly over-represented in lymphomas presenting outside of so-called "professional" lymphoid tissues, particularly in immune privilegedDLBCL. Mutational activation of TLR/MYD88 signaling endows lymphoma-initiating cells with a selective growth advantage at immune-privileged sites. The concomitant presence of $C D 79 B$ mutations, causing chronically active BCR signaling, may further promote the selective outgrowth of the tumor cells within these relatively stimulus-poor 


\section{CT MYD88 WT MYD88 L265P CT MYD88 WT MYD88 L265P}

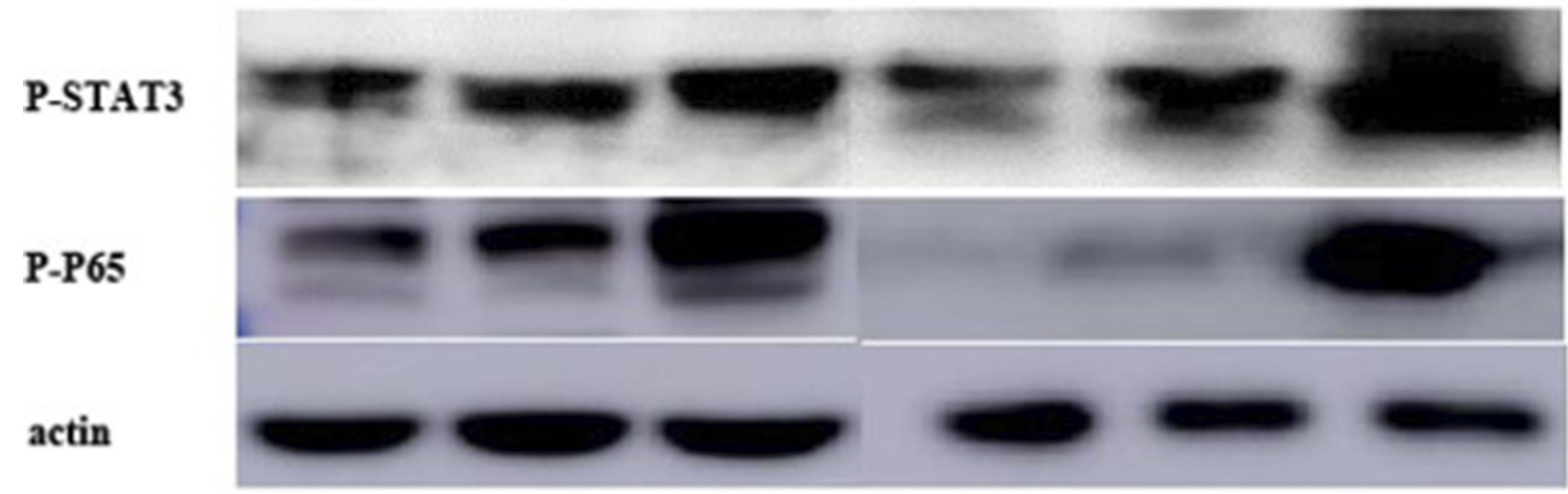

Figure 4 Western blot analysis of p-STAT3 and p-p65 protein expression in TMD8 and LYI cell lines harboring retroviral constructs for WT MYD88 or MYD88 mutant. The expression of P-STAT3 and P-p65 proteins were significantly upregulated in the MYD88 mutant group.

microenvironments. Taken together, these immunological sanctuaries could contain unique microenvironments that facilitate the migration, implantation, and/or growth of MYD88- and/or $C D 79 B$-mutated lymphoma cells, which would contribute to the homogeneity of testicular and central nervous system DLBCLs. ${ }^{34}$

In addition to the mutational status of MYD88, we also evaluated its copy number and protein expression. A previous study indicated that amplification of the MYD 88 locus is significantly more frequent in DLBCL cases with the MYD $88^{\mathrm{L} 265 \mathrm{P}}$ mutant than in those with MYD88 wild-type by array-based comparative genomic hybridization. ${ }^{15}$ However, our study showed that there was no significant difference in the amplification status between PT-DLBCL cases harboring the MYD88 wild type and L265P mutation. Thus, different methods may lead to different experimental results. We found that MYD 88 was overexpressed in $86.67 \%$ of PTDLBCL cases, which was higher than the rates reported by Choi et al. ${ }^{32}$ and Oishi et al. ${ }^{27}$ (38.7\% and 65\%, respectively). However, the amplification status was not associated with either the MYD 88 mutation frequency or its protein expression, which is in agreement with these studies. ${ }^{27,32}$ We also found that the expressional status of MYD88 was independent of its L265P mutation frequency and was significantly associated with tumor recurrence and a shortened diseasefree survival in patients with DLBCL. However, in the present study, neither MYD88 mutations nor its expressional status affected the prognosis of PT-DLBCL, consistent with the data of Oishi et al. ${ }^{27}$

\section{Conclusion}

Our results suggest that $M Y D 88$ and $C D 79 B$ mutations are important drivers of lymphomagenesis in PT-DLBCL. Thus, patients with PT-DLBCL may also benefit from therapy targeting MYD88 and/or BCR signaling for personalized treatment. This unique molecular and biological signature suggests that PT-DLBCL represents a unique subtype of DLBCL.

\section{Acknowledgments}

This study was supported by the Natural Science Foundation of Fujian Province (grant no. 2018J01277), Joint Funds for the Innovation of Science and Technology, Fujian Province (grant no. 2017Y9081), the Fujian Provincial Health Technology Project (grant nos. 2018-CX-10), and the Science and Technology Program of Fujian Province, China (grant no. 2018Y2003). We thank H. Nikki March, PhD, from Liwen Bianji, Edanz Editing China, for editing the English text of a draft of this manuscript.

\section{Disclosure}

The authors report no conflicts of interest in this work.

\section{References}

1. Duncan PR, Checa F, Gowing NF, et al. Extranodal non-Hodgkin's lymphoma presenting in the testicle: a clinical and pathologic study of 24 cases. Cancer. 1980;45:1578-1584. doi:10.1002/1097-0142(19800401)45:7<1578::AID-CNCR2820450711>3.0.CO;2-K 
2. Cheah CY, Wirth A, Seymour JF. Primary testicular lymphoma. Blood. 2014;123(4):486-493. doi:10.1182/blood-2013-10-530659

3. Zucca E, Roggero E, Bertoni F, et al. Primary extranodal nonhodgkin's lymphomas. Part 1. Gastrointestinal, cutaneous and genitourinary lymphomas. Ann Oncol. 1997;8:727-737. doi:10.1023/ A: 1008282818705

4. Lakhnana NK, Zafar $\mathrm{H}$, Tauseef $\mathrm{K}$, et al. Primary testicular lymphoma. Swiss Medical Weekly. 40(1):68. doi:10.1007/978-2-28792246-6 28.

5. Vitolo U, Ferreri AJM, Zucca E. Primary testicular lymphoma. Crit Rev Oncol Hemat. 2008;65(2):183-189. doi:10.1016/j.critrevonc.2007.08. 005

6. Ahmad SS, Idris SF, Follows GA, et al. Primary testicular lymphoma. Clin Oncol. 2012;24(5):358-365. doi:10.1016/j.clon.2012.02.005

7. Deng L, Xumonette ZY, Loghavi SN, et al. Primary testicular diffuse large B-cell lymphoma displays distinct clinical and biological features for treatment failure in rituximab era: a report from the International PTL Consortium. Leukemia. 2016;30(2):361-372. doi:10.1038/leu.2015.237

8. Baeuerle PA, Henkel T. Function and activation of NF-kappaB in the immune system. Annu Rev Immunol. 1994;12(1):141-179. doi:10.1146/annurev.iy.12.040194.001041

9. Perkins ND. The diverse and complex roles of NF-kappaB subunits in cancer. Nat Rev Cancer. 2012;12:121-132. doi:10.1038/nrc3204

10. Jost PJ, Ruland J. Aberrant NF-KB signaling in lymphoma: mechanisms, consequences, and therapeutic implications. Blood. 2007;109 (7):2700-2707. doi:10.1182/blood-2006-07-025809

11. Varettoni M, Arcaini L, Zibellini S, et al. Prevalence and clinical significance of the MYD88 (L265P) somatic mutation in Waldenström's macroglobulinemia and related lymphoid neoplasms. Blood. 2013;121(13):2522-2528. doi:10.1182/blood-2012-09-457101

12. Trøen G, Warsame A, Delabie J. CD79B and MYD88 mutations in splenic marginal zone lymphoma. ISRN Oncol. 2013;2012:252318. doi:10.1155/2013/252318

13. Puente XS, Pinyol M, Quesada V, et al. Whole-genome sequencing identifies recurrent mutations in chronic lymphocytic leukaemia. Nature. 2014;75:101-105. doi:10.1038/nature10113

14. Kim Y, Ju H, Kim DH, et al. CD79B and MYD88 mutations in diffuse large B-cell lymphoma. Hum Pathol. 2014;45(3):556-564. doi:10.1016/j.humpath.2013.10.023

15. Ngo VN, Young RM, Schmitz R, et al. Oncogenically active MYD88 mutations in human lymphoma. Nature. 2011;470:115-119. doi:10.1038/nature09671

16. Compagno M, Lim WK, Grunn A, et al. Mutations of multiple genes cause deregulation of NF- $\mathrm{KB}$ in diffuse large B-cell lymphoma. Nature. 2009;459(7247):717-721. doi:10.1038/nature07968

17. Alizadeh AA, Eisen MB, Davis RE, et al. Distinct types of diffuse large B-cell lymphoma identified by gene expression profiling. Nature. 2000;403:503-511. doi:10.1038/35000501

18. Kato M, Sanada M, Kato I, et al. Frequent inactivation of A20 in B-cell lymphomas. Nature. 2009;459:712-716. doi:10.1038/nature 07969

19. Davis RE, Ngo VN, Lenz G, et al. Chronic active B-cell-receptor signalling in diffuse large B-cell lymphoma. Nature. 2010;463 (7277):88-92. doi:10.1038/nature08638
20. Lenz G, Davis RE, Ngo VN, et al. Oncogenic CARD11 mutations in human diffuse large B cell lymphoma. Science. 2008;319 (5870):1676-1679. doi:10.1126/science.1153629

21. Pham-Ledard A, Cappellen D, Martinez F, et al. MYD88 somatic mutation is a genetic feature of primary cutaneous diffuse large B-cell lymphoma, leg type. J Invest Dermatol. 2012;132:2118-2120. doi:10.1038/jid.2012.102

22. Montesinos-Rongen M, Godlewska E, Brunn A, Wiestler OD, Siebert R, Deckert M. Activating L265P mutations of the MYD88 gene are common in primary central nervous system lymphoma. Acta Neuropathol. 2011;122:791-792. doi:10.1007/s00401-011-0891-2

23. Kraan W, Horlings HM, van Keimpema M, et al. High prevalence of oncogenic MYD88 and CD79B mutations in diffuse large B-cell lymphomas presenting at immune-privileged sites. Blood Cancer $J$. 2013;3(9):e139. doi:10.1038/bcj.2013.28

24. Lim KH, Yang Y, Staudt LM. Pathogenetic importance and therapeutic implications of NF-kappaB in lymphoid malignancies. Immunol Rev. 2012;246:359-378. doi:10.1111/j.1600-065X.2012.01105.x

25. Lim KH, Romero DL, Chaudhary D, Robinson SD, Staudt LM. IRAK4 kinase as a novel therapeutic target in the abc subtype of diffuse large B cell lymphoma. ASH Annu Meeting Abstr. 2012;120:62.

26. de Rooij MF, Kuil A, Geest CR, et al. The clinically active BTK inhibitor PCI-32765 targets B-cell receptor- and chemokine-controlled adhesion and migration in chronic lymphocytic leukemia. Blood. 2012;119:2590-2594. doi:10.1182/blood-2011-11-390989

27. Oishi N, Kondo T, Nakazawa T, et al. High prevalence of the MYD88 mutation in testicular lymphoma: immunohistochemical and genetic analyses. Pathol Int. 2015;65(10):528-535. doi:10.1111/pin.12336

28. Kraan W, van Keimpema M, Horlings HM, et al. High prevalence of oncogenic MYD88 and CD79B mutations in primary testicular diffuse large B-cell lymphoma. Leukemia. 2014;28(3):719-720. doi:10.1038/leu.2013.348

29. Zucca E. Patterns of outcome and prognostic factors in primary large-cell lymphoma of the testis in a survey by the international extranodal lymphoma study group. J Clin Oncol. 2003;21(1):20-27. doi:10.1200/JCO.2003.11.141

30. Gascoyne RD, Campo E, Jaffe ES, et al. Diffuse large Bcell lymphoma, not otherwise specified. In: Swerdlow SH, Campo E, Harris NL, et al. editors. WHO Classification of Tumours of Haematopoetic and Lymphoid Tissues In. Revised 4th. Lyon, France: International Agency for Research on Cancer (IARC); 2017:291-298.

31. Hans CP, Weisenburger DD, Greiner TC, et al. Confirmation of the molecular classification of diffuse large B-cell lymphoma by immunohistochemistry using a tissue microarray. Blood. 2004;103 (1):275-282. doi:10.1182/blood-2003-05-1545

32. Choi JW, Kim Y, Lee JH, et al. MYD88 expression and L265P mutation in diffuse large B-cell lymphoma. Hum Pathol. 2013;44 (7):1375-1381. doi:10.1016/j.humpath.2012.10.026

33. Young KH, Medeiros LJ, Chan WC. Diffuse large B-cell lymphoma. In: Orazi A, Weiss LM, Foucar K, Knowles DM, editors. Neoplastic Hematopathology. Philadelphia, PA, USA: Lippincott Willaims \& Wilkins; 2014:502-565.

34. Pals ST, de Gorter DJ, Spaargaren M. Lymphoma dissemination: the other face of lymphocyte homing. Blood. 2007;110:3102-3111. doi:10.1182/blood-2007-05-075176

OncoTargets and Therapy

Dovepress

\section{Publish your work in this journal}

OncoTargets and Therapy is an international, peer-reviewed, open access journal focusing on the pathological basis of all cancers, potential targets for therapy and treatment protocols employed to improve the management of cancer patients. The journal also focuses on the impact of management programs and new therapeutic

agents and protocols on patient perspectives such as quality of life, adherence and satisfaction. The manuscript management system is completely online and includes a very quick and fair peer-review system, which is all easy to use. Visit http://www.dovepress.com/ testimonials.php to read real quotes from published authors. 